\title{
EFFICACY OF INTERVENTIONS: AN EVIDENCE-BASED APPROACH
}

\section{Garth Alperstein \\ Community Paediatrician, Community Health Services, Central Sydney Area Health Service}

\section{Victor Nossar}

Service Director, Department of Community Paediatrics, South Western Sydney Area Health Service

$\mathrm{T}$ his article describes how an evidence-based approach was used to determine the most effective interventions to support the health of the children and adolescents of the Central Sydney Area.

Evidence-based medicine has been defined as 'the conscientious, explicit, and judicious use of current best evidence in making decisions about the care of individual patients'. ${ }^{1}$

The strategic plan Health gain for children and youth of Central Sydney ${ }^{2}$ sought to apply these concepts to interventions for populations rather than just for individuals. This approach has also been adopted by others, including the Canadian Task Force on the Periodic Health Examination and the Welsh Health Planning Forum. ${ }^{3,4}$ The Canadian task force encompassed the whole lifespan, and considered a number of child health issues. The Welsh group analysed child health issues more from the clinical perspective of hospital treatment and rehabilitation. The Central Sydney plan, however, focused more on population-based health promotion and prevention interventions for children and adolescents.

\section{THE PROCESS}

\section{Evidence-gathering}

Having described the health status of the children and young people in Central Sydney, the plan sought to identify interventions capable of addressing these health issues. Research was undertaken into available intervention strategies utilising the work of the abovementioned groups, MEDLINE searches, literature reviews and consultation with expert advisers for each of the identified issues. It was sought to establish, from available evidence:

- what is known to work

- what is known not to work

- unproven strategies or conflicting findings.

\section{Evidence-rating}

Recommendations were then made about each intervention, using an established rating scale, ${ }^{3}$ which assesses efficacy on the basis of best available evidence and grades interventions accordingly. The grades are:

A good evidence to support implementation

B fair evidence to support implementation

C inconclusive evidence to support implementation or abandon intervention
D fair evidence to abandon intervention

E good evidence to abandon intervention.

These ratings were awarded after analysis of the quality of the available evidence, taking into consideration the methodology described in the identified studies and any advice from relevant experts. The quality of the evidence was assessed according to the following criteria:

I at least one properly randomised controlled trial

II-1 well-designed controlled trials without randomisation

II-2 well-designed cohort or case-control studies, preferably from more than one centre

II-3 comparisons of times or places with or without interventions

III opinions of respected authorities, based on clinical experience, descriptive studies or reports of expert committees.

Generally, the strongest recommendations (a rating of A or E) were reserved for interventions whose efficacy was supported or negated by high-quality evidence (I or II-1). Interventions for which there was evidence assessed as II-2 and II-3 were generally awarded a rating of B or D. Where there was limited evidence to either support or negate an intervention or strategy, the $\mathrm{C}$ rating was assigned to that intervention.

\section{Review of interventions}

All of the available health interventions or strategies were also reviewed to determine whether they formed a part of current services, offered by Central Sydney Area Health Service (CSAHS) or by other organisations within Central Sydney. The interventions were then categorised as either 'yes', 'no' or 'partial', in regard to whether a particular intervention was being implemented in Central Sydney.

In an attempt to further define the optimal approach to achieve the desired child health outcomes, each strategy was then reassessed in light of its assigned rating and the quality of evidence supporting it, alongside formal consideration of its implementation status. Taking all these factors into account, an estimation was then made about whether there existed an opportunity for either health gain or for reorientation of that service.

\section{Examples}

For example, if a particular strategy was assigned a high rating (A or B) and sound methodology had been used in the supporting studies identified (I, II-1), and, as well, if the strategy or activity was not being implemented at that time by CSAHS or the other services in Central Sydney, then an opportunity for health gain would be created from the implementation of that strategy (for example, a comprehensive home visiting program). 


\section{COCHRANE COLLABORATION}

Conversely, if there was good evidence that a strategy had low efficacy or was ineffectual, and that activity was being implemented at that time by CSAHS or other services, this would indicate that there was an opportunity to reorient the resources being used by that service (for example, distraction hearing testing of all seven-month-old babies).

\section{CONCLUSION}

In the final analysis, it was recognised that not all interventions lent themselves to this type of evidencebased evaluation (especially not the interventions of a community development type). The relevance and importance of these kinds of interventions need to be assessed by other means than the application of this kind of evidence-based methodology. As Sackett stated, 'evidence based medicine is not restricted to randomised controlled trials and meta-analyses. It involves tracking down the best external evidence with which to answer the clinical question'. ${ }^{1}$ He particularly cautioned against purchasers and managers using evidence-based medicine as a means to cut health care costs. In fact, the adoption of the most efficacious interventions to maximise both quality and quantity of health outcomes could increase costs.

This process has proved to be useful in identifying areas of practice where changes in services could achieve improved health, as well as highlighting those areas of practice where additional strategies or services are required but may not yet be in place.

\section{REFERENCES}

1. Sackett DL, Rosenberg, MWC, Gray JAM, Haynes RB, Richardson WS. Evidence based medicine: what it is and what it isn't. BMJ 1996; 312: 71-72.

2. Alperstein G, Thomson J, Crawford J. Health gain for children and youth of Central Sydney. Strategic plan. Sydney: Central Sydney Area Health Service, NSW Health Department, 1996. ISBN 0731300297.

3. Canadian Task Force on the Periodic Health Examination, Minister of Supply and Services Canada. Canadian guide to clinical preventive health care. Ottawa: Canada Communication Group Publishing, 1994.

4. Welsh Health Planning Forum. Protocol for investment in health gain. Maternal and early child health. Cardiff: Welsh Office NHS Directorate, 1991. it

\section{HEALTH GAIN FOR THE CHILDREN AND YOUTH OF CENTRAL SYDNEY}

continued from p. 108

3. Welsh Health Planning Forum. Protocol for investment in health gain. Maternal and early child health. Cardiff: Welsh Office NHS Directorate, 1991.

4. Alperstein G, Thomson J, Crawford J. Health gain for children and youth of Central Sydney. Strategic plan. Sydney: Central Sydney Area Health Service, NSW Health Department, 1996. ISBN 0731300297.

\section{Katrina Williams}

Paediatrician, Clinical Epidemiology Unit, New

Children's Hospital, Westmead

The Cochrane Collaboration was established in 1993, with the aim of preparing, maintaining and disseminating systematic reviews of the effectiveness of health care. It is expected that systematic reviews of the available evidence will become the first step in creating policies, changing practice and developing appropriate future research.

Development of the collaboration has demanded the personal and financial commitment of large numbers of individuals (clinicians, academics, consumers and policy makers) and organisations (governments, research agencies and charitable institutions) internationally. Currently the Cochrane database collects systematic reviews of randomised and controlled trials.

\section{THE CHILD HEALTH FIELD}

A child health field was proposed at the 4th International Cochrane Colloquium because of a perceived need for more reviews in the area of child health.

The aim of the child health field is to promote the interests of children within the collaboration by undertaking reviews that address questions that are important for clinicians and parents in a form that is easily translated into policy and practice. Activities include promoting and publicising the field to relevant professionals, consumers and professional bodies. A child health field database of references is currently being developed.

While the Cochrane centres are responsible for training, the child health field will also take on a content-relevant training role. In Australia, training has already been established through the Centre for Community Child Health \& Ambulatory Paediatrics in conjunction with the Department of Biostatistics and Epidemiology of the University of Melbourne, and so far has been provided at the Royal Children's Hospital in Melbourne. In addition, those in the child health field have identified areas within child health where existing review groups will not serve required needs. A planning meeting for a review group on developmental, psychosocial and learning problems was held in 1997 in an attempt to fill one such area of need.

\section{HOW TO BECOME INVOLVED?}

Clinicians can become involved in the Cochrane effort in two ways: by becoming users of reviews on clinically relevant areas or by undertaking reviews themselves. To undertake a review requires training, as well as consultation with the review group most relevant to the chosen topic. A commitment must be made to update the review on a yearly basis, for life. This may sound onerous, but in reality, it can be passed on to another reviewer. It is expected that, in future, review group administrators will look for any new trials on topics registered with them and pass them directly to the reviewers. 